\title{
Gluon mass at finite temperature in Landau gauge
}

\section{Pedro Bicudo*}

CFTP, Instituto Superior Técnico, CFTP, Universidade de Lisboa, 1049-001, Lisboa, Portugal E-mail: bicudo@tecnico.ulisboa.pt

\section{Orlando Oliveira}

CFC, Departamento de Física, Faculdade de Ciências e Tecnologia, Universidade de Coimbra, 3004-516 Coimbra, Portugal

E-mail: orlandodfis.uc.pt

\section{Paulo J. Silva}

CFC, Departamento de Física, Faculdade de Ciências e Tecnologia, Universidade de Coimbra, 3004-516 Coimbra, Portugal

E-mail: psilva@teor.fis.uc.pt

\section{Nuno Cardoso}

CFTP, Instituto Superior Técnico, CFTP, Universidade de Lisboa, 1049-001, Lisboa, Portugal E-mail: nunocardosodcftp.ist.utl.pt

Using lattice results for the Landau gauge gluon propagator at finite temperature, we investigate its interpretation as a massive type bosonic propagator. In particular, we estimate a gluon mass from Yukawa-like fits to the lattice data and study its temperature dependence.

31st International Symposium on Lattice Field Theory - LATTICE 2013

July 29 - August 3, 2013

Mainz, Germany

\footnotetext{
${ }^{*}$ Speaker.
} 

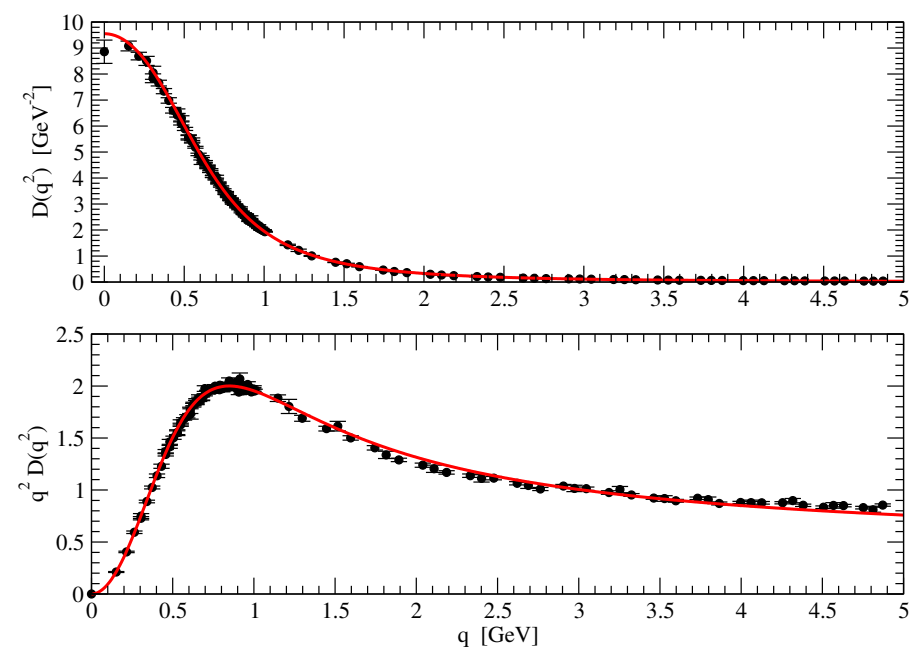

Figure 1: Landau gauge gluon propagator at $\beta=6.0$, renormalized at $\mu=3 \mathrm{GeV}$, combining $32^{4}, 48^{4}, 64^{4}, 80^{4}$ data.

\section{Introduction}

Lattice QCD not only is quite important to compare QCD with experiment, but also is ideal to test theories, approximations and models. Here we address pure gauge theory near the phase transition region.

At $T=0$, pure gauge $\mathrm{SU}(3) \mathrm{QCD}$ exhibits color screening and flux tubes [1,2,3], while at large $T$ Debye screening occurs [4]. At $T=T_{c} \sim 270 \mathrm{MeV}$, there is evidence of a finite gluon mass scale in the $\pi$ and $K$ multiplicities in heavy ions [5].

Here we complement the outstanding study [6] of the gluon masses in SU(2) for $2 T_{c}<T<$ $15000 T_{c}$. We study [7] the finite temperature range $T<2 T_{c}$ in SU(3) pure gauge theory.

The study of the gluon propagator and gluon mass require gauge fixing, and we resort to Landau gauge.

\section{Gluon propagator with Landau gauge fixing at $\mathbf{T}=0$}

On the lattice, Landau gauge fixing is applied to a configuration $U_{\mu}(\mathbf{x})$ by maximizing the function,

$$
F_{U}[g]=C_{F} \sum_{\mathbf{x}, \mu} \operatorname{Re}\left\{\operatorname{Tr}\left[g(\mathbf{x}) U_{\mu}(\mathbf{x}) g^{\dagger}(\mathbf{x}+\hat{\mu})\right]\right\},
$$

where $g(\mathbf{x})$ is a gauge transformation. The maximization procedure leads to $\partial_{\mu} A_{\mu}^{a}=0$. We apply a (Fourier accelerated) Steepest Descent method. We have tested this method both in CPU's and GPU's $[8,9]$.

We compute the $D\left(p^{2}\right)$, shown in Fig. 1, with pure gauge lattice simulations, utilizing the Wilson action for pure gluons, and the expectation value,

$$
\left\langle A_{\mu}^{a}(p) A_{v}^{b}(p)\right\rangle=V \delta(p-k) \delta^{a b}\left(\delta_{\mu v}-\frac{p_{\mu} p_{v}}{p^{2}}\right) D\left(p^{2}\right)
$$




\begin{tabular}{cccccc}
\hline Temp. $(\mathrm{MeV})$ & $\beta$ & $L_{s}$ & $L_{t}$ & $\mathrm{a}[\mathrm{fm}]$ & $1 / \mathrm{a}(\mathrm{GeV})$ \\
\hline 121 & 6.0000 & 64 & 16 & 0.1016 & 1.9426 \\
162 & 6.0000 & 64 & 12 & 0.1016 & 1.9426 \\
194 & 6.0000 & 64 & 10 & 0.1016 & 1.9426 \\
243 & 6.0000 & 64 & 8 & 0.1016 & 1.9426 \\
260 & 6.0347 & 68 & 8 & 0.09502 & 2.0767 \\
265 & 5.8876 & 52 & 6 & 0.1243 & 1.5881 \\
275 & 6.0684 & 72 & 8 & 0.08974 & 2.1989 \\
285 & 5.9266 & 56 & 6 & 0.1154 & 1.7103 \\
290 & 6.1009 & 76 & 8 & 0.08502 & 2.3211 \\
305 & 6.1326 & 80 & 8 & 0.08077 & 2.4432 \\
324 & 6.0000 & 64 & 6 & 0.1016 & 1.9426 \\
366 & 6.0684 & 72 & 6 & 0.08974 & 2.1989 \\
397 & 5.8876 & 52 & 4 & 0.1243 & 1.5881 \\
428 & 5.9266 & 56 & 4 & 0.1154 & 1.7103 \\
458 & 5.9640 & 60 & 4 & 0.1077 & 1.8324 \\
486 & 6.0000 & 64 & 4 & 0.1016 & 1.9426 \\
\hline
\end{tabular}

Table 1: Lattice setup used for the computation of the gluon propagator at finite temperature. The $\beta$ was adjusted to have $L_{s} a \simeq 6.5 \mathrm{fm}$. Simulations have been done in Milipeia and Centaurus clusters at the University of Coimbra, using Chroma and PFFT libraries.

We utilize sufficiently large volumes $V=L_{s}{ }^{4}$, since a larger volume implies we can reach smaller infrared (IR) momenta for the computation of $D\left(p^{2}\right)$. We also use small lattice spacing, to reduce the $\mathscr{O}\left(a^{2}\right)$ corrections effects, relevant both in the IR and medium momentum range [10].

In the ultraviolet (UV), we find the propagator is massless and similar to the 1-loop predictions.

In the IR the propagator is compatible with a massive denominator and the simplest fit is a Yukawa [2] up to $p \approx 600 \mathrm{MeV}$

$$
M_{g}=648(7) \mathrm{MeV},
$$

or a rational function with complex conjugate poles

$$
M_{g}=626 \pm i 362 \mathrm{MeV} \text {. }
$$

Moreover we also apply the more elaborate fit of a running gluon mass, [2]

$$
D\left(p^{2}\right)=\frac{Z\left(p^{2}\right)}{p^{2}+M^{2}\left(p^{2}\right)} .
$$

The running gluon mass is fitted with a parameter $m_{0}=723(11) \mathrm{MeV}$

$$
M^{2}\left(p^{2}\right)=\frac{m_{0}^{4}}{p^{2}+m_{0}^{2}}, \quad Z\left(p^{2}\right)=\frac{z_{0}}{\left[\log \frac{p^{2}+r m_{0}^{2}}{\Lambda^{2}}\right]^{\gamma}},
$$

and it works up to $p=4.1 \mathrm{GeV}$. This ansatz has a similar functional form to the decoupling solution of the Dyson-Schwinger equations, and to the prediction of the Refined-Zwanziger action [11]. 

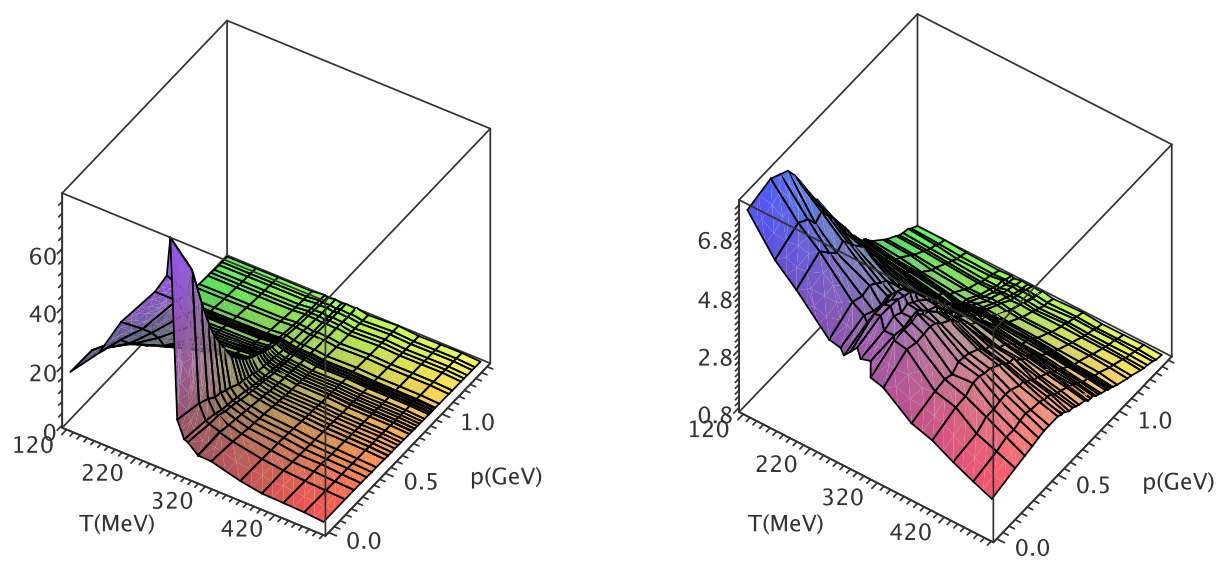

Figure 2: 3D plots momenta and temperatures of the longitudinal and transverse propagators.

\section{Gluon propagator at $T>0$}

At finite $T$, we project the Lorentz structure of the propagator $D_{\mu v}^{a b}(\hat{q})$ with two independent form factors,

$$
D_{\mu \nu}^{a b}(\hat{q})=\delta^{a b}\left(P_{\mu \nu}^{T} D_{T}\left(q_{4}^{2}, \vec{q}\right)+P_{\mu \nu}^{L} D_{L}\left(q_{4}^{2}, \vec{q}\right)\right) ;
$$

for details on the definition of the transverse $\left(D_{T}\right)$ and longitudinal $\left(D_{L}\right)$ components see [7].

On the lattice, finite temperature $T=\frac{1}{a L_{t}}$ is simply introduced by reducing the extent of temporal direction $L_{t}<<L_{s}$. Note that we utilize the same large volume with $L_{s} \sim 6.5 \mathrm{fm}$ for all $T-$ see table 1. Moreover, all lattice data is renormalized fitting the momenta in the UV region to the 1-loop inspired propagator,

$$
D_{\text {lattice }}\left(q^{2}\right)=\frac{K}{q^{2}}\left(\ln \frac{q^{2}}{\Lambda^{2}}\right)^{-13 / 22}
$$

The renormalization constants are computed such that $D\left(q^{2}\right)=Z_{R} D_{\text {lattice }}\left(q^{2}\right)$ after requiring the renormalization propagator to verify $D\left(\mu^{2}\right)=1 / \mu^{2}$ with $\mu=4 \mathrm{GeV}$. $D_{T}$ and $D_{L}$ are renormalised independently but we observe $Z_{L}$ and $Z_{T}$ differ by less than $2 \%$.

\section{Gluon mass at finite $T$}

We plot the inverse of the finite T propagators in Fig. 3. For IR momenta, they are again compatible with a massive denominator. Notice $D_{L}^{-1}$ is linear in the infrared, while $D_{T}^{-1}$ bends. In the UV the propagators have a logarithmic behavior, $D_{i}^{-1} \sim \log$.

The simplest ansatz for a massive propagator is,

$$
D(p)=\frac{1}{p^{2}+M^{2}}, \Rightarrow M=1 / \sqrt{D(0)} .
$$



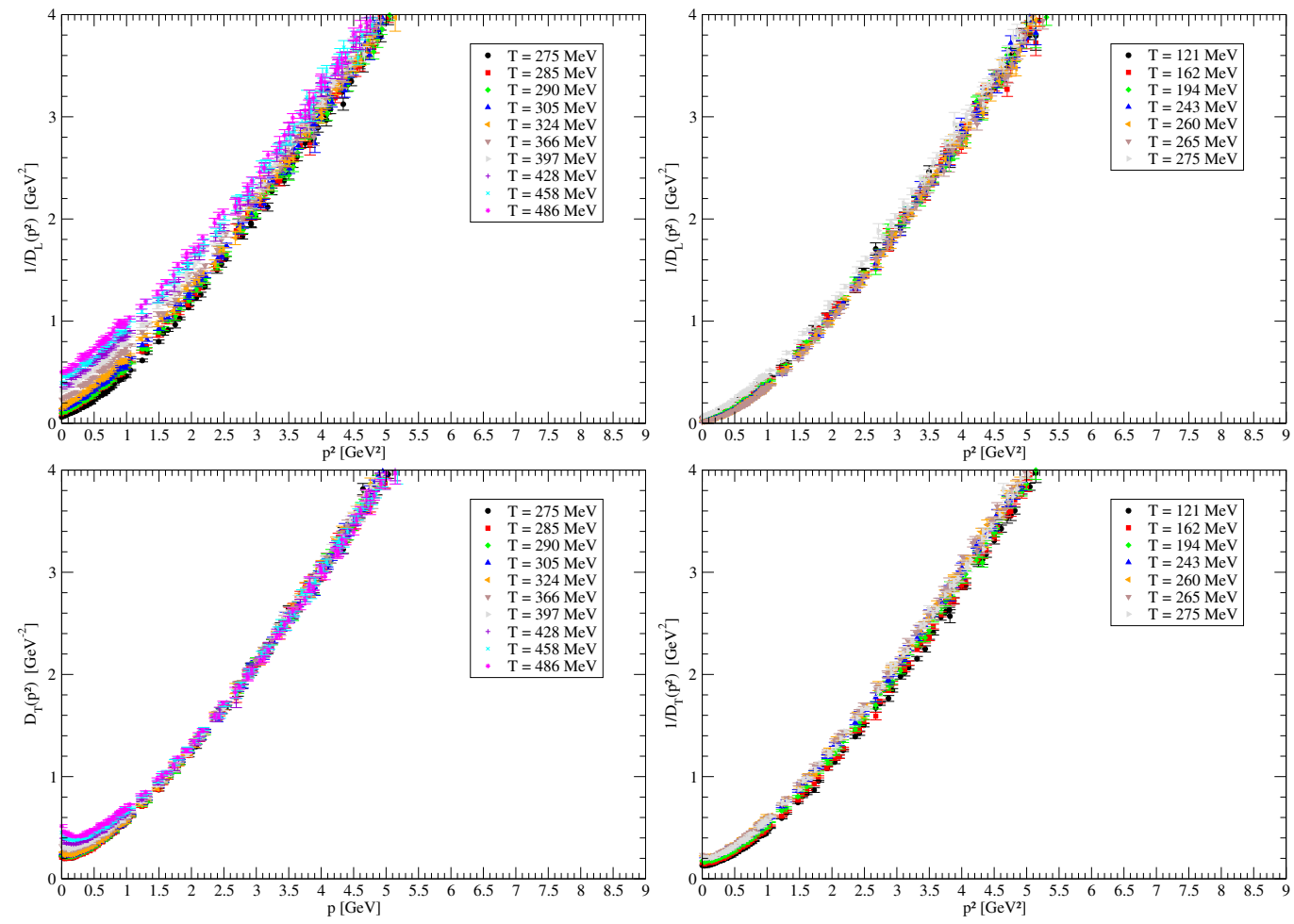

Figure 3: Inverse of the longitudinal and transverse propagators at different finite temperatures $T$.

The values for $1 / \sqrt{D(0)}$ are shown in Fig. 4. Close to $T_{c}, D_{L}$ clearly signals the transition, while $D_{T}$ is aparently flat. At $T \sim 2 T_{c}$, the two masses cross, $M_{L} \sim M_{T}$.

We also consider a better ansatz, adequate for IR momenta, by fitting $D_{i}$ to a Yukawa with mass $m$,

$$
D_{i}\left(p^{2}\right)=\frac{Z}{p^{2}+m^{2}}
$$

and look for the largest fitting range $p_{\max }$. While this fits quite well $D_{L}$, the Yukawa ansatz does not fit $D_{T}$. In Fig. 5 we show the fit of the mass $m$ and of the factor $Z$. While $Z$ peaks at the transition, the mass $m$ is minimum but clearly finite.

\section{Conclusion}

We compute the gluon propagator in Landau gauge Lattice QCD at finite $0<T<2 T_{c}$. The longitudinal component $D_{L}$ is peaked at $T=T_{c}$. In the infrared, we fit $D_{i}$ with massive Yukawa ansatze, the fit to $D_{L}$ is more stable than the fit to $D_{T}$. The fitted longitudinal gluon mass $M_{L}$ is compatible with confinement screening at $T \sim 0 . M_{L}$ is also consistent with Debye screening at $T>>0$, We observe $M_{L}$ is minimum at $T \sim T_{c}$, but finite [7] as suggested by multiplicites of $\pi$ and $k$ production in heavy ion collisions. 


\begin{tabular}{ccccc}
\hline$T$ & $p_{\max }$ & $Z_{L}$ & $M_{L}$ & $\chi^{2} /$ d.o.f. \\
\hline 121 & 0.467 & $4.28(16)$ & $0.468(13)$ & 1.91 \\
162 & 0.570 & $4.252(89)$ & $0.3695(73)$ & 1.66 \\
194 & 0.330 & $5.84(50)$ & $0.381(22)$ & 0.72 \\
243 & 0.330 & $8.07(67)$ & $0.374(21)$ & 0.27 \\
260 & 0.271 & $8.73(86)$ & $0.371(25)$ & 0.03 \\
265 & 0.332 & $7.34(45)$ & $0.301(14)$ & 1.03 \\
275 & 0.635 & $3.294(65)$ & $0.4386(83)$ & 1.64 \\
285 & 0.542 & $3.12(12)$ & $0.548(16)$ & 0.76 \\
290 & 0.690 & $2.705(50)$ & $0.5095(85)$ & 1.40 \\
305 & 0.606 & $2.737(80)$ & $0.5900(32)$ & 1.30 \\
324 & 0.870 & $2.168(24)$ & $0.5656(63)$ & 1.36 \\
366 & 0.716 & $2.242(55)$ & $0.708(13)$ & 1.80 \\
397 & 0.896 & $2.058(34)$ & $0.795(11)$ & 1.03 \\
428 & 1.112 & $1.927(24)$ & $0.8220(89)$ & 1.30 \\
458 & 0.935 & $1.967(37)$ & $0.905(13)$ & 1.45 \\
486 & 1.214 & $1.847(24)$ & $0.9285(97)$ & 1.55 \\
\hline
\end{tabular}

Table 2: Mass $M_{L}$ and factor $Z_{L}$ parameters of the Yukawa fits to the longitudinal propagators at finite $T$.

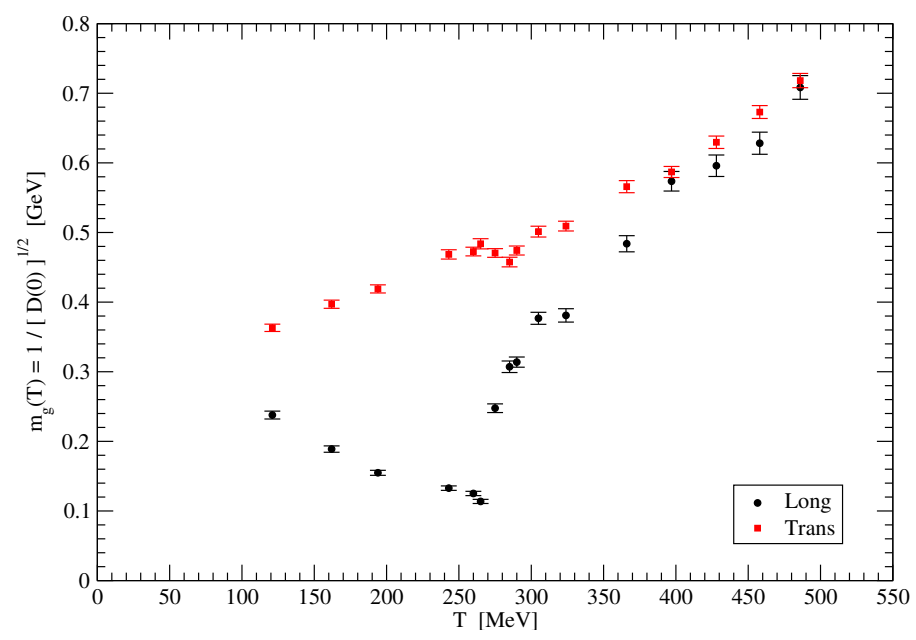

Figure 4: Longitudinal and transverse masses fitted with the simplest ansatz at finite temperatures $T$.

\section{Acknowledgments}

P. J. Silva acknowledges support by F.C.T. under contract SFRH/BPD/40998/2007. Work supported by projects CERN/FP/123612/2011, CERN/FP/123620/2011 and PTDC/FIS/100968/2008, projects developed under initiative QREN financed by UE/FEDER through Programme COMPETE. 


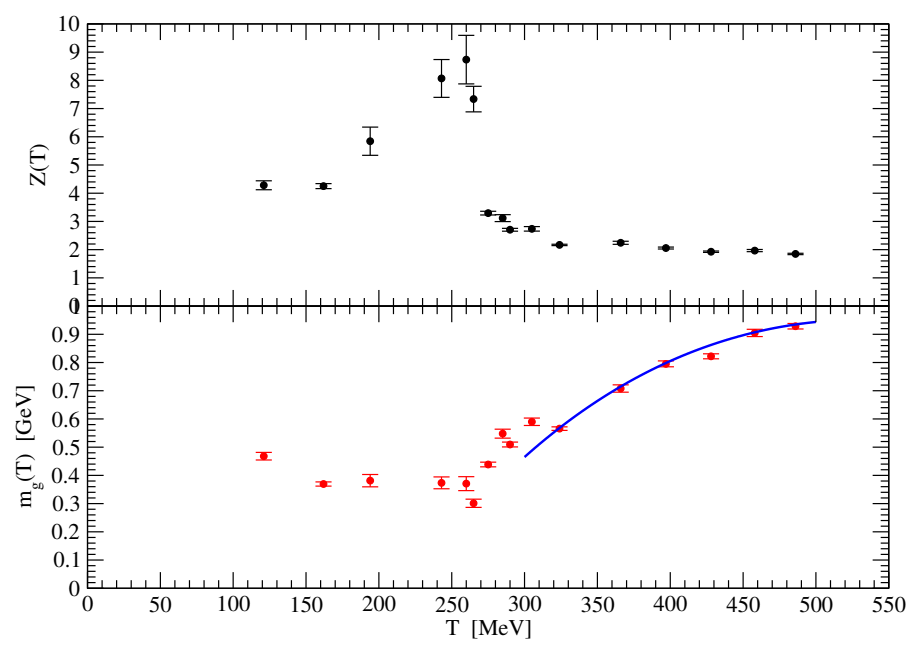

Figure 5: Mass and factor parameters fitted with the Yukawa ansatz at finite temperatures $T$.

\section{References}

[1] J. M. Cornwall, Phys. Rev. D 26, 1453 (1982).

[2] O. Oliveira and P. Bicudo, J. Phys. G 38, 045003 (2011) [arXiv:1002.4151 [hep-lat]].

[3] N. Cardoso, M. Cardoso and P. Bicudo, Phys. Rev. D 88, 054504 (2013) [arXiv:1302.3633 [hep-lat]].

[4] M. Doring, K. Huebner, O. Kaczmarek and F. Karsch, Phys. Rev. D 75, 054504 (2007) [hep-lat/0702009 [HEP-LAT]].

[5] P. Bicudo, F. Giacosa and E. Seel, Phys. Rev. C 86, 034907 (2012) [arXiv:1202.1640 [hep-ph]].

[6] U. M. Heller, F. Karsch and J. Rank, Phys. Rev. D 57, 1438 (1998) [hep-lat/9710033].

[7] P. J. Silva, O. Oliveira, P. Bicudo and N. Cardoso, arXiv:1310.5629 [hep-lat].

[8] N. Cardoso, P. J. Silva, P. Bicudo and O. Oliveira, Comput. Phys. Commun. 184, 124 (2013) [arXiv:1206.0675 [hep-lat]].

[9] M. Schroeck and H. Vogt, Comput. Phys. Commun. 184, 1907 (2013) [arXiv:1212.5221 [hep-lat]].

[10] O. Oliveira and P. J. Silva, Phys. Rev. D 86, 114513 (2012) [arXiv:1207.3029 [hep-lat]].

[11] D. Dudal, O. Oliveira and N. Vandersickel, Phys. Rev. D 81, 074505 (2010) [arXiv:1002.2374 [hep-lat]].

[12] A. Maas, J. M. Pawlowski, L. von Smekal and D. Spielmann, Phys. Rev. D 85, 034037 (2012) [arXiv:1110.6340 [hep-lat]].

[13] R. Aouane, V. G. Bornyakov, E. M. Ilgenfritz, V. K. Mitrjushkin, M. Muller-Preussker and A. Sternbeck, Phys. Rev. D 85, 034501 (2012) [arXiv:1108.1735 [hep-lat]]. 